Reprinted from MONTHLY WeAther Review, Vol. 118, No. 11, November 1990

American Meteorotapical Society

$-44-47$

$7 / 230$

\title{
The 27-28 October 1986 FIRE IFO Cirrus Case Study: Cloud Optical Properties Determined by High Spectral Resolution Lidar
}

\author{
C. J. GRund AND E. W. Eloranta \\ University of Wisconsin-Madison. Deparment of Meteorology, Madison. Wisconsin
}

(Manuscript received 17 February 1989, in final form 12 June 1990)

\begin{abstract}
During the FIRE cirrus IFO, the High Spectral Resolution Lidar (HSRL) was operated from a roof top site on the University of Wisconsin-Madison campus. Because the HSRL technique separately measures the molecular and cloud particle backscatter components of the lidar return, the optical thickness is determined independent of particle backscatter. This is accomplished by comparing the known molecular density distribution to the observed decrease in molecular backscatter signal with altitude. The particle to molecular backscatter ratio yields calibrated measurements of backscatter cross section that can be plotted to reveal cloud morphology without distortion due to attenuation. Changes in cloud particle size shape and phase affect the backscatter to extinction ratio (backscatter-phase function). The HSRL independently measures cloud particle backscatter phase function. This paper presents a quantitative analysis of the HSRL cirrus cloud data acquired over an $\sim 33$ hour period of continuous near-zenith observations. Correlations between small-scale wind structure and cirrus cloud morphology have been observed. These correlations can bias the range averaging inherent in wind profiling lidars of modest vertical resolution, leading to increased measurement errors at cirnus altitudes. Extended periods of low intensity backscatter were noted between more strongly organized cirrus cloud activity. Optical thicknesses ranging from $0.01-1.4$, backscatter-phase functions between $0.02-0.065 \mathrm{sr}^{-1}$, and backscatter cross sections spanning 4 orders of magnitude were observed. The altitude relationship between cloud top and bottom boundaries and the cloud optical center altitude was dependent on the type of formation observed. Cirrus features were observed with characteristic wind drift estimated horizontal sizes of $5 \mathrm{~km}-400 \mathrm{~km}$. The clouds frequently exhibited cellular structure with vertical to horizontal dimension ratios of 1:5-1:1.
\end{abstract}

\section{Introduction}

Determinations of the optical properties, structure, and the vertical and horizontal extent of cirrus clouds have broad applications in remote sensing and the atmospheric sciences. Cirrus clouds reflect incoming solar radiation and trap outgoing terrestrial radiation; thus, the global energy balance depends upon the optical and morphological characteristics of these clouds. Scattering and absorption by cirrus clouds affect measurements made by many satellite-borne and groundbased remote sensors. Scattering of ambient light by the cloud, and thermal emissions from the cloud, can increase measurement background noise. Multiple scattering processes can adversely affect the divergence of optical beams propagating through these clouds.

Predicting the effects of greenhouse gasses, aerosols, albedo changes, and solar fluctuations on climate requires the development of models that accurately account for the highly variable, nonlinear influence of clouds on radiative balance. Models of the feedback mechanisms between cirrus clouds and earth's climate

Corresponding author address: Dr. Christian J. Grund, Department of Meteorology, University of Wisconsin-Madison, Madison, WI 53706. can be tested and improved by studying time series of cloud formation, maintenance, and dissipation processes. Good models require good initialization data and must generate realistic cloud radiative properties. Spatial and temporal histories of the optical and morphological characteristics of real clouds are required to fulfill these needs.

Because of its precise ranging capabilities, spatial resolution, and sensitivity, lidar has played an important role in the detection, depiction, and characterization of cirrus clouds (Evans et al. 1966; Platt et al. 1987; Sassen et al. 1990), and for the verification of cloud heights derived from satellite-borne sensor measurements (Wylie and Menzel 1989). Lidar systems that make one measurement at each range can adequately determine backscatter intensity distributions for optically thin clouds; however, they cannot independently determine absolute optical quantities. As optical thickness increases, simple plots of lidar backscatter intensity may produce a distorted representation of cloud morphology, and can produce serious errors in cloud altitude determinations. This is because the lidar return signal from any range depends on both the backscatter cross section and the optical depth to that range. Single-channel lidar systems may not separately measure backscatter and extinction. In order to pro- 
duce calibrated measurements, single-channel lidar retrieval techniques must be constrained by additional information or assumptions about the optical properties or distribution of the scatterers (Spinhime et al. 1980; Klett 1981; Weinman 1984; Eloranta and Forrest 1986). Direct measurements require the determination of a signal intensity calibration at each range.

The High Spectral Resolution Lidar (HSRL) has been specifically designed (Shipley et al. 1983; Sroga et al. 1983) to produce calibrated measurements of aerosol and cloud particle optical depth, extinction corrected cloud morphology, and backscatter phase function. The HSRL spectrally separates molecular backscatter from cloud particle and aerosol backscatter. This is possible because thermal agitation Doppler broadens the backscattered spectrum from molecules. Small aerosols and cloud particles that contribute significantly to the backscatter are more massive than molecules and thus exhibit slower Brownian drift velocities that produce insignificant broadening of the scattered spectrum. Using the known distribution of molecular scattering cross section to provide a calibration reference at every range, extinction is unambiguously determined from the observed range dependent decrease in molecular backscatter intensity. The ratio of measured aerosol to molecular backscatter intensity provides the aerosol backscatter cross section at each range. Because aerosol and gaseous absorption are negligible at the HSRL wavelength, the backscatter phase function can be directly measured. A more detailed description of the HSRL theory and the definitions of lidar measured quantities may be found in the Appendix.

HSRL measurements of cirrus cloud optical properties were first acquired (Grund 1987) during the FIRE Intensive Field Observations (Starr 1987). In this paper we discuss the application of the HSRL to cirrus cloud measurements and provide a comprehensive survey of the cirrus cloud optical properties determined during the case study period.

\section{System characteristics}

Using a multietalon pressure-tuned Fabry-Perot spectrometer, the HSRL simultaneously observes the lidar return in two channels. The spectrally narrow "aerosol channel," centered on the transmitted wavelength, is most sensitive to aerosol scattering and to the central region of the Doppler-broadened molecular spectrum. With a prominent notch in the center of its bandpass, the spectrally wider "molecular channel" accepts the entire Doppler-broadened molecular spectrum, while rejecting much of the aerosol scatter. Thus, the signal in each channel represents a different linear combination of the aerosol and molecular scattering contributions to the lidar return. Complete separation of the two channel signals requires the determination of a $2 \times 2$ matrix of inversion coefficients. The two coefficients, which represent the aerosol backscatter transfer function for each channel, are determined by diffusely filling the receiver telescope with attenuated laser light and observing the response of the two channel signals to a spectral scan of the receiver (Grund 1987). The coefficients that represent the molecular backscatter transfer function for each channel are calculated from the calibration scan convoluted with a model of the molecular backscatter spectrum (Yip and Nelkin 1964), corrected for temperature and pressure at each altitude. Table 1 summarizes the HSRL system characteristics at the time the case study data was acquired.

Data acquisition and control of the multietalon spectrometer and scanning mirror system are accomplished by a DEC $11 / 73$ computer running a multiuser operating system. Real time and time-averaged display of range square and log corrected data is provided by an in A-scope. Data are acquired in 4 second "shots" of 32000 accumulated, range-resolved lidar profiles. Each shot is written to magnetic tape for later processing.

The tapes are subsequently written to a $\mathbf{2 . 6}$ gigabyte capacity write once optical disk, which facilitates repeated access to individual segments of large datasets. Calibration and data analysis are performed on a VAX 11/751 computer, which has on-line image display and graphics capability.

TABLE 1. Summary of HSRL operating parameters during the 1986 FIRE cirrus IFO. Because of the narrow spectral bandwidth. narrow field of view, high repetition rate, and photon counting de tection scheme, the HSRL was capable of measuring cirrus cloud optical properties under day or night conditions, while maintaining eye-safe operations.

\begin{tabular}{|c|c|c|}
\hline \multicolumn{3}{|c|}{ HSRL Receiver } \\
\hline Telescope: & $\begin{array}{l}\text { Primary diameter } \\
\text { Secondary diameter } \\
\text { Focal length } \\
\text { F.O.V. (full width) }\end{array}$ & $\begin{array}{l}.35 \mathrm{~m} \\
.114 \mathrm{~m} \\
3.85 \mathrm{~m} \\
320 \mathrm{mR}\end{array}$ \\
\hline Interference filter. & FWHM & $1 \mathrm{~nm}$ at $510.6 \mathrm{~nm}$ \\
\hline Pre-filter etalons: & $\begin{array}{l}\text { Plate diameter } \\
\text { Etalon spacers } \\
\text { Combined FWHM }\end{array}$ & $\begin{array}{l}50 \mathrm{~mm} \\
1.003 . .726 \mathrm{~mm} \\
2.5 \mathrm{pm}\end{array}$ \\
\hline High resolution etalon: & $\begin{array}{l}\text { Plate diameter } \\
\text { Etalon spacer } \\
\text { Bandwidth (FWHM) }\end{array}$ & $\begin{array}{l}150 \mathrm{~mm} \\
12.786 \mathrm{~mm} \\
.6 \mathrm{pm}\end{array}$ \\
\hline Signal detection: & Photon counting & $>10 \mathrm{mHz}$ \\
\hline Photomultiplier tubes: & $\begin{array}{l}\text { EMI Gencom 9863B/100 } \\
\text { HSRL Transmitter }\end{array}$ & low afterpuising \\
\hline & $\begin{array}{l}\text { Laser, Wavelength } \\
\text { Transmitted beam } \\
\text { diameter }\end{array}$ & $\begin{array}{l}\mathrm{CuCl}_{2}, 510.6 \mathrm{~nm} \\
3 \mathrm{um}\end{array}$ \\
\hline & $\begin{array}{l}\text { Transmitted power } \\
\text { Bandwidth (FWHM) } \\
\text { Pulse repetition rate } \\
\text { Pulse length }\end{array}$ & $\begin{array}{l}50 \mathrm{~mW} \text { at } 510.6 \mathrm{~nm} \\
.4 \mathrm{pm} \\
8 \mathrm{kHz} \\
15 \mathrm{~ns}\end{array}$ \\
\hline
\end{tabular}




\section{Data analysis}

In order to facilitate interpretation of the HSRL measurements by a varied community of cirrus researchers, we have chosen two forms for the representation of our data for this case study. The first, greyscale imagery of the uninverted "aerosol channel" backscatter intensity, has the advantage of higher temporal resolution, but lacks a calibrated correction for extinction with range. The second data representation format, as contour plots of calibrated backscatter cross section, reveals the true distribution of backscattered intensity, but lacks the ability to depict the fine structural detail of the cirrus clouds. This resolution limitation was imposed by the low average power of the laser transmitter that necessitated time averaging to mitigate the effects of statistical noise in the inverted signals. We have chosen to reserve detailed calibrated analysis for this paper while presenting the more qualitative grey-scale imagery in the companion lidar intercomparison paper (Sassen et al. 1990).

Although the HSRL was primarily designed for the measurement of boundary layer aerosol properties, it has been successfully adapted to the task of cirrus cloud characterization (Grund 1987). Several difficulties arise when assessing cirnus cloud optical properties with the HSRL:

a) the signals are reduced by the additional range to the cirrus clouds (up to $15 \mathrm{~km}$ at zenith in midlatitudes);

b) the molecular scattering intensity is reduced by the lower air density at cirrus altitudes;

c) low temperatures at cirrus altitudes decreace the Doppler width of the scattered molecular spectrum making accurate separation from the unbroadened particulate scattering more difficult;

d) cloud backscatter intensities can be very large compared to molecular scattering thus requiring extreme accuracy $(\sim 0.1 \%)$ in the determination of the inversion coefficients; and

e) during daylight operations cirrus clouds scatter sunlight into the receiver field of view, increasing the background-light-induced noise. Aerosol scattering cross sections observed in previous operations rarely achieved even thin cirrus scattering cross section values.

To combat these problems, and still maintain reasonable calibration accuracy, time resolution, and measurement linearity, the following data processing algorithm has been applied:

a) A series of inverted molecular and aerosol profiles is generated with $\sim 4 \mathrm{~s}$ time resolution. Because drifts in the system bandpass and receiver-transmitter tuning have nonlinear effects on the relative transmission of the receiver channels, a new set of separation coefficients is chosen for each of these profiles by the following method:
1) A synthetic calibration scan is produced for each shot by a linear interpolation in time between the adjacent calibration scans.

2) The spectral offset between the center of the receiver bandpass and the transmitted wavelength is estimated from observations of the ratio of the aerosol to molecular channel signals under uniform aperture illumination with transmitted laser light. This ratio is measured at $\sim 2$ min intervals during operations. The ratio is estimated for each shot time by an interpolating spline fit to the observations. Since the receiver-transmitter may exhibit relative drift of either sign, and the bandpass characteristics have a slight asymmetry, the sense of the tuning offset is assumed to be the same as the overall drift observed between the preceding and subsequent calibration scans. With the tuning drift determined, the inversion coefficients are calculated from the synthetic calibration scan and a model of the molecular scattering spectrum that includes the effects of Brillouin scattering.

b) Because the temporal and spatial distribution of extinction is highly variable in cirrus clouds, statistical fluctuations in lidar signals cannot be reduced by straight forward block averaging of profiles. The reason for this is evident in Eq. (A I). Because the signal from each range depends on an exponential term that varies from profile to profile, simple sums will exhibit a range dependent bias toward the shots with least attenuation (Milton and Woods 1987). To minimize this effect, inverted shots are initially summed only to the extent that the backscatter profile can be qualitatively discerned from noise (typically $\sim 30 \mathrm{~s}$ ). Subjective groups of these intermediate sums are formed by adding range resolved profiles together, as long as the backscatter profiles do not appear markedly different. Optical properties are determined from each of these grouped profiles, then combined in a time-weighted average to achieve the reported time resolution.

c) In calculating the optical properties, the inverted molecular signal has not been applied directly within the cirrus clouds. Instead, the HSRL molecular channel signal has been smoothed according to the following algorithm:

1) Regions dominated by Rayleigh scattering are determined both above and below the cloud (this is possible because first-cut HSRL backscatter cross section profiles clearly indicate regions of enhanced particulate scatter, even in the presence of a small crosstalk term ).

2) In these regions, a least squares fit is produced from the observed separated molecular signal to the expected profile for a pure molecular scattering atmosphere calculated from a radiosonde profile of pressure and temperature. This is acceptable since the extinction contribution from particles is small and most 
of the signal slope in the separated molecular profile is associated with the known decrease in density with height.

3) The clear-air observed signals are replaced with the smooth best fit estimates above and below the cloud.

4) The cloud optical thickness is determined from the decrease in the best fit molecular signal across the cloud determined in step 3, while accounting for the expected decrease in molecular cross section with altitude.

5) The vertical profile of extinction within the cloud is calculated from the separated particulate backscatter profile using a Bernoulli solution constrained by the optical thickness determined in step 4 and the assumption of a constant backscatter to extinction ratio. The Bernoulli technique is employed in this solution to reduce the logarithmic range derivative of the lidar Eq. (A1) to a first order equation which can be solved explicitly for the extinction profile, given the total optical thickness and an assumed power law relationship between backscatter and extinction (Weinman 1988).

6) The in-cloud molecular backscatter signal is replaced with a smooth estimate calculated from the extinction profile determined in step 5 and the known altitude distribution of the molecular scattering cross section $\beta_{m}(R)$ [see Eq. (A3)]. In this way, noise is removed from the molecular scattering profile, while the spatial distribution of extinction is closely maintained. Backscatter cross sections [Eq. (AS)] are calculated using this estimate of $P_{m}(R)$.

7) Backscatter phase functions are reported as bulk quantities calculated over the entire depth of cloud because the Bernoulli solution employed to mitigate the effects of noise assumes the backscatter to extinction ratio is constant.

Penetration of clouds, hence cloud-top altitudes, are assured in HSRL data by the presence of molecular backscatter signal from above the cloud. Molecular signal was clearly evident within the reported time resolutions throughout this case study period, suggesting confidence in the cloud-top altitudes indicated at the level of the minimum plotted backscatter cross section contours. Errors in optical thickness and backscatter phase function were determined by estimating the effects of photon counting statistics on both the range resolved signals and the uncorrelated background signal according to the methods detailed in Grund (1987).

Cirrus can sometimes form plate-like crystals that fall flat-face-down because of aerodynamics. Consequently, the HSRL was operated $3^{\circ}-5^{\circ}$ from zenith in order to minimize the impact of specular backscatter on the measurements of backscatter phase function and backscatter cross section. Specular backscatter enhancements by a factor of at least 13 have been observed, and larger enhancements seem possible. The $e^{-1}$ full width of the forward scatter diffraction peak was $\sim 1.5^{\circ}$ (at $\left.1.06 \mu \mathrm{m}\right)$ suggesting a minimum crystal diameter of $\sim 50 \mu \mathrm{m}$. Fortunately, at our operating wavelength, $\mathrm{Ci}$ size parameters are fairly large. This reduces the angular width of the diffraction peak so that the enhanced backscatter drops off rapidly with declination from zenith.

\section{Lidar derived optical properties}

Several periods of cirrus exhibiting differing optical and morphological characteristics were observed during the case study period. We will begin with a discussion of optically thin subvisual cirrus, proceed to a quantitative description of a mesoscale uncinus complex, continue with a comparison of altocumulus and ice cloud scattering properties, and conclude with the characterization of the optically thicker cirrus layer. Throughout this discussion we will refer to the optical properties defined in the Appendix. In addition, the reported optical thicknesses $(\tau)$ have had the effects of molecular extinction removed so that they represent only the attenuation due to particle scattering. Likewise, backscatter cross sections represent aerosol scattering quantities without molecular scattering contributions, and will be denoted $\beta_{a \pi} / 4 \pi$. Because we frequently observe correlations between small-scale wind features and lidar backscatter, isotachs are included on each backscatter cross section plot for reference. For a pictorial overview of the HSRL observations, the reader is directed to the lidar intercomparison paper elsewhere in this volume (Sassen et al. 1990).

\section{a. Subvisible and background cirrus}

The occurrence of subvisible cirrus has been a topic of interest in the last few years because of the potential effects on IR wavelength remote sensors. In addition to the attenuation provided by the cloud, thermal emissions from the cloud and ambient light scattered by the cloud can contribute to measurement background noise. Even tenuous clouds can develop significant optical thickness when probed by shallow angle or horizontally viewing long range sensors. In addition, low-level aerosol backscatter can complicate the retrieval of optical depth from simple lidar observations because calibrations often rely upon the determination of regions characterized by pure molecular scattering. Cloud visibility is a complicated property that depends on many factors including contrast, discernible structure, and sun angle in addition to optical thickness. However, is this paper, we will adopt the convention of Sassen et al. (1989) and consider clouds with a zenith angle $\tau \leqslant 0.03$ as subvisible. Within this case stidy period, several subvisible clouds were observed by the HSRL.

Figure 1 shows a contour plot of the $\beta_{a r} / 4 \pi$ of an isolated subvisible cirrus cloud. The contour interval 


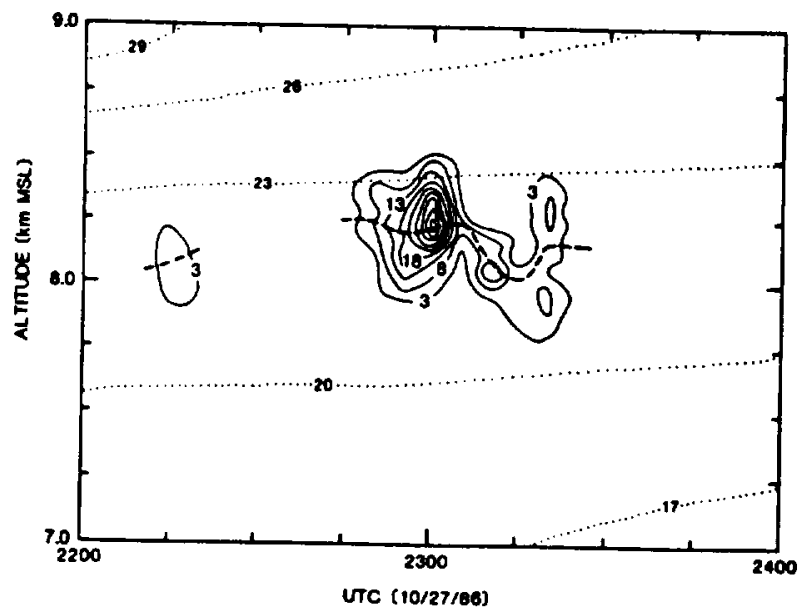

FIG. 1. Contour plot of the backscatter cross section $\left(10^{-4} \mathrm{~km}^{-1}\right.$ $\mathbf{s s}^{-1}$ in solid line) of a subvisible cirrus cloud. The dashed line indicates the optical midcloud height (see text). The average optical thickness of this cloud between 2245 and 2320 UTC was 0.03 with an average bulk backscatter phase function of $0.028 \mathrm{sr}^{-1}$. Isotachs (dotted line) are plotted in $\mathrm{m} \mathrm{s}^{-1}$ from the Fort McCoy radiosondes launched at 2100 and 0000 UTC.

is $5.0(10)^{-4} \mathrm{~km}^{-1} \mathrm{sr}^{-1}$ starting from $3.0(10)^{-4} \mathrm{~km}^{-1}$ $\mathrm{sr}^{-1}$. The dashed line marks the optical midaltitude of the cloud; half the cloud optical depth is accumulated to either side of this line. This height was determined from the Bernoulli solution for the in-cloud extinction profile. The maximum value of $\beta_{a \pi} / 4 \pi$ measured in this cloud was $4.1(10)^{-3} \mathrm{~km}^{-1} \mathrm{sr}^{-1}$ at 2301 UTC. Assuming negligible temporal evolution, translation with the $25 \mathrm{~m} \mathrm{~s}^{-1}$ ambient wind suggests a horizontal extent of $\sim 60 \mathrm{~km}$ for this cloud. When the $2245-2322$ data are averaged together, the mean optical thickness for this cloud was determined to be $0.03 \pm 0.006$. The bulk-average backscatter phase function for this cloud was found to be $0.028 \pm 0.007 \mathrm{sr}^{-1}$. Backscatter intensification near 2300,2310 , and 2320 at $\sim 8 \mathrm{~km}$ suggests this cirrus cloud was composed of $\sim 5 \mathrm{~km}$ wide shallow cells or bands spaced $\sim 20 \mathrm{~km}$ apart, imbedded in a less dense background cloud.

On two occasions during the case study, we have observed extended periods of enhanced backscatter with little apparent structure. Because the HSRL technique does not distinguish between backscatter from small aerosols (e.g., dust, $\mathrm{H}_{2} \mathrm{SO}_{4}$ droplets, etc.) and backscatter from cloud particles, we cannot be certain that these subvisible background veils are cirrus; however, because more dense cirrus are observed at the subvisible veil altitudes both before and after these observations, these features appear to be at least associated with cirrus. Regardless of particle identification, the existence of extended regions of elevated scatter at these altitudes have similar impacts on radiative balance and the operation of remote sensors.

A persistent region of enhanced backscatter of this type is evident in Fig. 2 from $8.5-13.8 \mathrm{~km}$, and a two layer formation apparent in Fig. 3 between 7.0 and $13.8 \mathrm{~km}$. Because of the small backscatter signals from these clouds, the range resolution has been degraded to $\sim 900 \mathrm{~m}$ and temporal resolution has been reduced to 30-60 minutes. In higher time and spatial resolution images of the raw lidar data, these veils seem to be rather stable and are not seen to exhibit the cellular structure present in the subvisible cirrus of the type shown in Fig. 1. It is clear from these data that average backscatter cross sections of $<1(10)^{-4} \mathrm{~km}^{-1} \mathrm{sr}^{-1}$ can exist over extended regions even when structured clouds are not detected. Evidently, there must be several mechanisms for the generation and maintenance of subvisible clouds.

Because radiosonde humidity measurements do not often extend above $\sim 10 \mathrm{~km}$ and are frequently unreliable at these altitudes, it is difficult to suggest particle composition from the availability of moisture for the formation of ice particles in these clouds. However, radiosondes launched from Platteville at 0000 and 0300 show consistently moist ( $\mathrm{RH}$ with respect to ice $>70 \%$ ) regions from $8.8 \mathrm{~km}-9.5 \mathrm{~km}$ and $8.2 \mathrm{~km}-10.0 \mathrm{~km}$, respectively. The 0900 and 1200 soundings indicated moist layers $7.2 \mathrm{~km}-10.2 \mathrm{~km}$ and $6.6 \mathrm{~km}-10.1 \mathrm{~km}$, respectively, both with maxima $\mathrm{RH}$ with respect to ice in excess of $100 \%$.

Between 0000 and 0400 , the subvisible cloud exhibited an average optical thickness of $0.01 \pm 0.004$. From 0920 to 1140 the average optical thickness was 0.003 \pm 0.002 . Wind-drift estimates of the size of the features

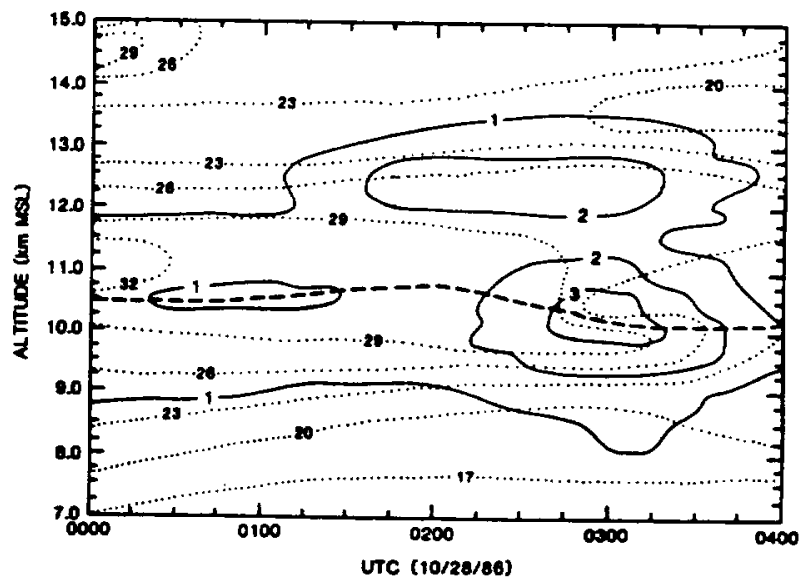

Fig. 2. Background veils of enhanced particle backscatter are frequently observed at cirrus altitudes. The average optical thickness of this layer was $\sim 0.01$. Backscatter cross section contours (solid line) are in units of $10^{-4} \mathrm{~km}^{-1} \mathrm{sr}^{-1}$, optical midcloud heights are plotted in dashed line, and isotachs are plotted in dotted line in $\mathrm{m} \mathrm{s}^{-1}$ from the Fort McCoy radiosondes launched at 0000,0300 and 0900 UTC (the 0600 radiosonde was not launched). Note the correlation between wind speed and backscatter cross section contour patterns. Correla. tions between wind velocity and backscatter distribution (e.g., 1000 UTC between 9 and $10.5 \mathrm{~km}$ ) can bias wind profiles acquired from future space based Doppler lidars with insufficient vertical resolution (see text). 


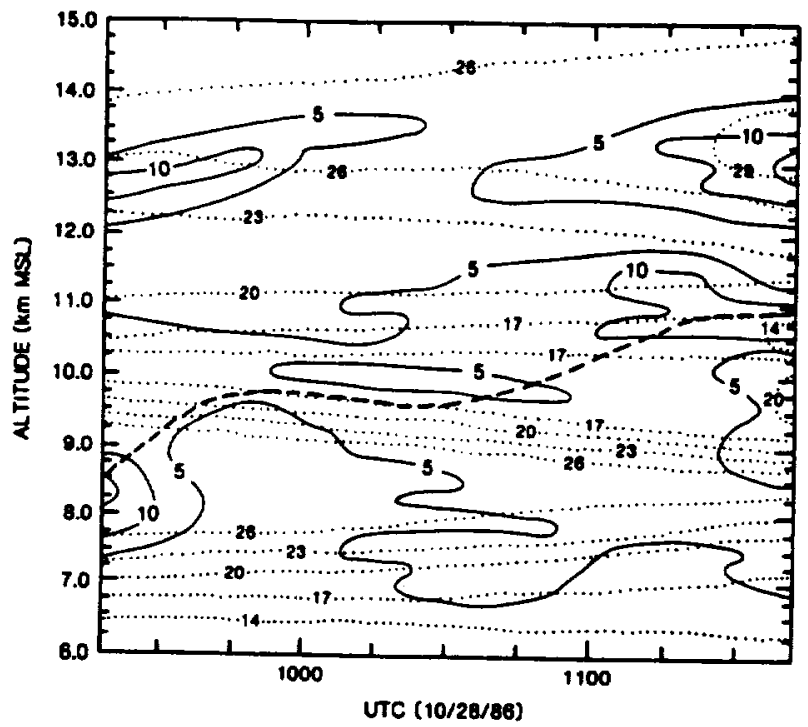

Fig. 3. A two-layer region of enhanced backscatter. Backscatter cross section contours (solid line) are in units of $10^{-5} \mathrm{~km}^{-1} \mathrm{sr}^{-1}$, optical midcloud heights are plotted in dashed line, and wind speeds are plotted in dotted line in $\mathrm{m} \mathrm{s}^{-1}$ from the For McCoy radiosondes launched at 0900 and 1200 UTC. Backscatter cross section values are $\sim$ half of those shown in Fig. 2, and although the wind profiles are quite different, correlations between wind and backscatter are still evident.

depicted in Figs. 2 and 3 suggest that they extend for more than $400 \mathrm{~km}$ and $180 \mathrm{~km}$, respectively. One implication of such large-scale optically thin clouds is that remote sensors attempting to view horizontally within such layers could easily encounter significant optical thickness, even though the cloud may not produce a visual manifestation. Another implication of such extensive cloud blankets is they can alter earth's radiative balance, if present on a global scale, while going undetected by space-borne passive remote sensors.

Isotachs plotted in Fig. 2 show a general correlation between wind speed maxima and backscatter cross section contours. Note the 26 and $29 \mathrm{~m} \mathrm{~s}^{-1}$ contours closely follow the pattern of backscatter cross section distribution. These apparent pattern correlations have been observed in several cases, though a consistent set of correlation characteristics has not been established. It is not clear whether the sheer is part of the cirrus generation mechanism or if cirrus formation and winds are both responding to the same environmental forcing.

Because wind-profiling Doppler lidars rely on backscatter from naturally occurring aerosols, correlations between wind sheer and the distribution of backscatter can bias vertically-averaged wind measurements of insufficient range resolution. As an example, a wind profiling lidar observing the 0200 profile between 7.5 and $14.5 \mathrm{~km}$ with $1 \mathrm{~km}$ resolution would produce an rms wind speed error of $\sim 0.4 \mathrm{~m} \mathrm{~s}^{-1}$ due solely to range averaging with the maximum error of $-1.2 \mathrm{~m} \mathrm{~s}^{-1} \mathrm{oc}-$ curring at $9.8 \mathrm{~km}$. When weighted by the backscatter cross section at each range, the $1 \mathrm{~km}$ averaged wind speed estimates produced rms errors exceeding $0.8 \mathrm{~m}$ $\mathrm{s}^{-1}$ with a peak error of $2.4 \mathrm{~m} \mathrm{~s}^{-1}$ at $8.6 \mathrm{~km}$.

Figure 3 shows another example of a strong correlation between wind sheer and backscatter cross section. Note the packing of isotachs between 9.5 and $10.5 \mathrm{~km}$ coincident with the thin cloud region at 1000 UTC. When subjected to the above wind profiling lidar analysis, this data produced rms errors of $\sim 0.5 \mathrm{~m} \mathrm{~s}^{-1}$ with a peak error of $1.7 \mathrm{~m} \mathrm{~s}^{-1}$ at $9.8 \mathrm{~km}$ due solely to the $1 \mathrm{~km}$ range averaging of the wind speeds between 6.5 and $14.5 \mathrm{~km}$. Backscatter weighting of the range average produced an rms wind speed error of $\sim 1.0 \mathrm{~m}$ $\mathrm{s}^{-1}$ with a peak error of $-4.1 \mathrm{~m} \mathrm{~s}^{-1}$ at $9.0 \mathrm{~km}$. A study of the effects of velocity-backscatter correlations and resolution specifications on wind profiling lidar measurements is reported in Grund et al. (1990).

\section{b. Mesoscale uncinus complex (MUC)}

Figure 4 presents a contour map of the absolute backscatter cross section from a mesoscale uncinus complex (MUC) observed between 0500 and 0900 UTC 28 October. For the basic structural description and a pictorial view of the relative backscatter from this mesoscale uncinus complex refer to Sassen et al. (1990). A series of uncinus generating cells is evident between 9.5 and $11.0 \mathrm{~km}$, particularly between 0530 and 0700 . Each of these cells is about $150 \mathrm{~m}$ thick. Cloud translation with the $10 \mathrm{~km}$ wind $(20 \pm 10 \mathrm{~m}$ $\mathrm{s}^{-1}$ ) would suggest the cells are $\sim 4-12 \mathrm{~km}$ across; thus, they have a height to width aspect ratio in the range $\sim$ 1:50-1:180. Averaging times of $\sim 12$ minutes were chosen for each profile in this cross section so that ex-

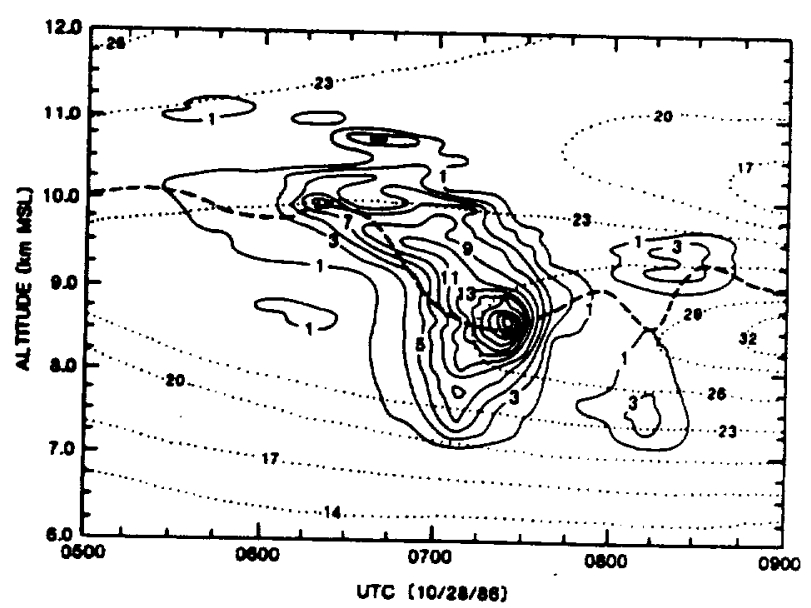

FIG. 4. Cin : backscatter cross section $\left(10^{-3} \mathrm{~km}^{-1} \mathrm{sr}^{-1}, \longrightarrow\right)$ and optical midcloud height (- - ) of a mesoscale uncinus complex. The average optical thickness of this system be. . . . .0600 and 0750 UTC was $0.58 \pm 0.05$ which varied from $0.09 \pm 0.03$ at $0750 \mathrm{~L}$ IC to $1.1 \pm 0.3$ at 0718 UTC. The bulk backscatter phase function averaged over the same time period was $0.042 \pm 0.015 \mathrm{sr}^{-1}$. The MUC passed over Madison just ahead of a mesoscale wind jet. Again note the apparent association of wind speed $\left(\mathrm{m} \mathrm{s}^{-1}, \ldots\right)$ ) with cirrus morphology. Isotachs were determined from the Fort McCoy radio-
sondes launched at 0300 and 0900 UTC. 
pected signal-noise-induced errors in the average cloudbackscatter cross section were limited to $\pm 15 \%$. The maximum $\beta_{a x} / 4 \pi$ for this MUC was determined to be $0.024 \mathrm{~km}^{-1} \mathrm{sr}^{-1}$ at 0722 near $8.7 \mathrm{~km}$ altitude. preceding the passage of a wind jet maximum of $\sim 34 \mathrm{~m} \mathrm{~s}^{-1}$. Both the Ft. McCoy and Platteville soundings at 0300 and 0900 ( 0600 was not acquired) indicate the regions above $7.4 \mathrm{~km}$ were consistently moist ( $>70 \% \mathrm{RH}$ with respect to ice ) and occasionally reached supersaturation as high as $\sim 108 \%$ with respect to ice. The radiosonde profiles also show an abrupt decrease in relative humidity below $7.4 \mathrm{~km}$ suggesting the steep contour gradient at 0700 at $7.2 \mathrm{~km}$ altitude is related to rapid ice crystal evaporation in the dry environment beneath the complex. Analysis of the profles averaged over the 0600-0750 period indicates this system had a mean optical thickness of $0.58 \pm 0.05$, which varied from $0.09 \pm 0.03$ at 0750 to $1.1 \pm 0.3$ at 0718 . The bulk backscatter phase function averaged over the same time period was $0.042 \pm 0.015 \mathrm{sr}^{-1}$.

Figures 5, 6, and 7 are the 0600,0700 , and 0800 GOES IR images covering the IFO study area that show the MUC passing directly over Madison (stationary white-on-black square in south-central Wisconsin). The relatively bright (cold) complex is embedded in a less intense eastward moving cloud band that extends $\sim$ NW-SE from a more extensive cloud shield covering northern Wisconsin. Cloud image tracking indicates the band is moving eastward at $\sim 23 \mathrm{~m} \mathrm{~s}^{-1}$, close to the wind-drift velocity at cirrus altitudes. The complex seems to be propagating southeastward as the band moves east. Radiosonde data show the $8.5 \mathrm{~km}$ wind backing from $\sim 300^{\circ}$ to $\sim 270^{\circ}$ as the complex passes; however, the winds show a slight but consistent veering with height above $8 \mathrm{~km}$ throughout this period. Figure 4 indicates the most optically dense cloud regions were near $8.5 \mathrm{~km}$. Thus, the apparent southeastward prop-

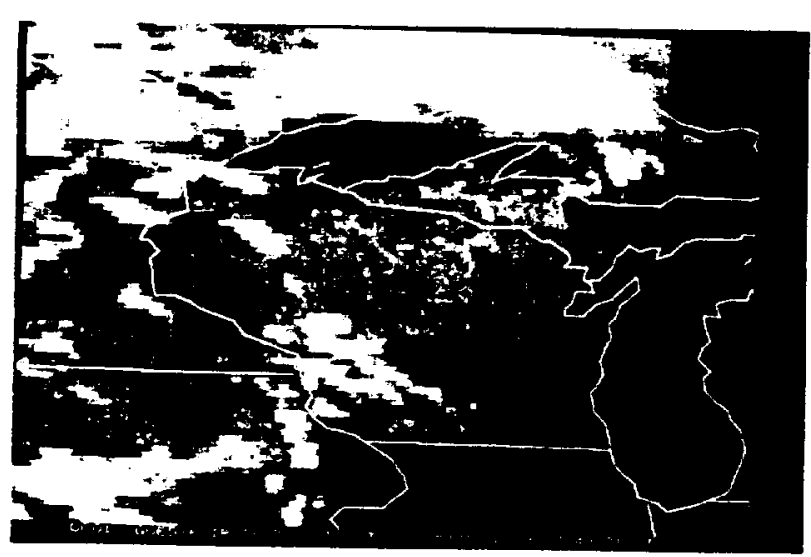

FIG. 5. The 0600 UTC GOES IR image showing the cirrus cloud band over Madison (white pixel in south-central Wisconsin) extending NW to SE from a larger cirrus shield covering northern WI. The relatively bright cloud regions just to the NW of Madison moved rapidly to the SE along the cloud band over the next two hours (see Fig. 6).

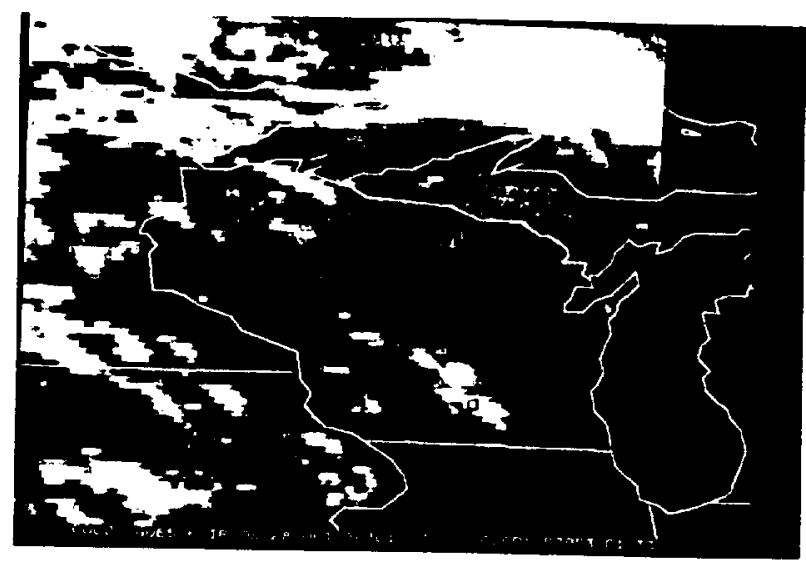

FIG. 6. The 0700 UTC GOES IR image shows the mesoscale uncinus complex (MUC) passing directly over the lidar site as it propagates southeastward along the eastward moving cloud band. The lidar time height cross section through the cloud band and MUC as it passed overhead was shown in Fig. 4.

agation of the complex seems to be a displacement of the MUC generating region along the west wind-driven cloud band, rather than a translation of the complex with the ambient wind.

Isotachs, interpreted from the 0300 and $0900 \mathrm{Ft}$. McCoy radiosonde data, show an apparent relationship between wind speeds and the MUC backscatter cross section distribution. Note the close proximity in time and altitude between the wind speed maximum and the backscatter cross section maximum. Also note that the cloud bottom occurs where the time height cross section indicates relatively steady winds. Further, the decrease in cloud top altitude seems to be related to the development of a wind minimum at $10.5 \mathrm{~km}$. The jet and the wind minimum are of smaller scale than

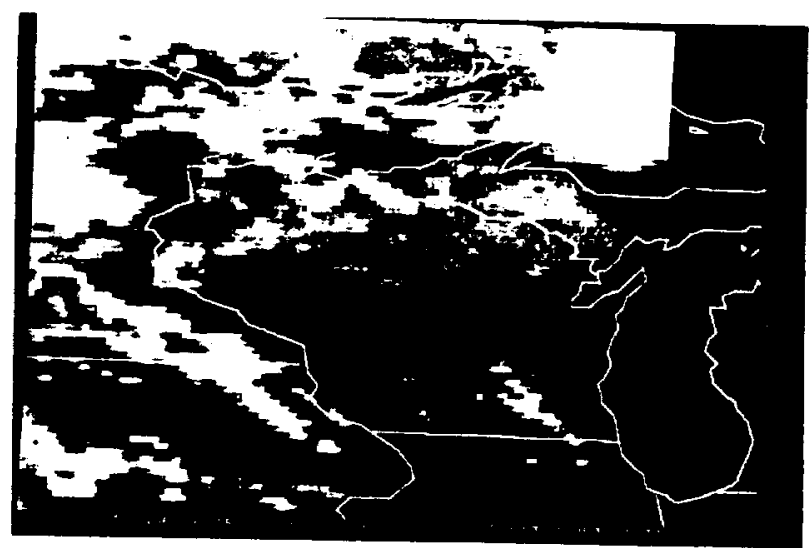

FIG. 7. The 0800 UTC GOES IR image shows the apparent dissipation of the MUC as it continues to propagate to the SE along the cloud band. The lidar ( see Fig. 4) shows the backscatter structure of the cloud band following the MUC. Displacement estimates from Figs. 5-7 suggest the cloud band is moving eastward at $\sim 23 \mathrm{~m} \mathrm{~s}^{-1}$, in agreement with rawinsonde-determined winds at the lidar-determined cirrus altitudes. 
the FIRE IFO radiosonde network. They appear strongly only in the Ft. McCoy soundings, and are just discernible in the Platteville sounding data; thus, no attempt has been made to interpolate the radiosonde data to Madison observation times. Future observations of this type would benefit from a more dense temporal and spatial net of wind observations.

The MUC observations also demonstrate the limitations inherent in interpreting cirrus cloud morphology strictly from zenith time height cross section measurements. If the wind sheer is deduced from the apparent slope of the virga trails, the wind maximum would be expected near the upper cloud level at $\sim 10$ $\mathrm{km}$. In fact, the wind contours indicate the maximum of $\sim 35 \mathrm{~m} \mathrm{~s}^{-1}$ near $8.5 \mathrm{~km}$, decreasing to $\sim 18 \mathrm{~m} \mathrm{~s}^{-1}$ at $10 \mathrm{~km}$. Clearly, the time-height cross section of this complex does not represent a stationary phenomena translating with the ambient winds. Rather, in addition to translation, the clouds are undergoing significant evolution. Cloud genesis is probably related to circulations about the mesoscale jet, and the apparent shape of virga may be partially governed by the local distribution of moisture. Clearly, serious study of the morphology of such mesoscale phenomena can greatly benefit from the acquisition of real time three-dimensional lidar observations.

\section{c. Altocumulus versus cirrus}

From 1200 to 1500 UTC 28 October, a two-level cirrus-altocumulus formation was observed. Figure 8

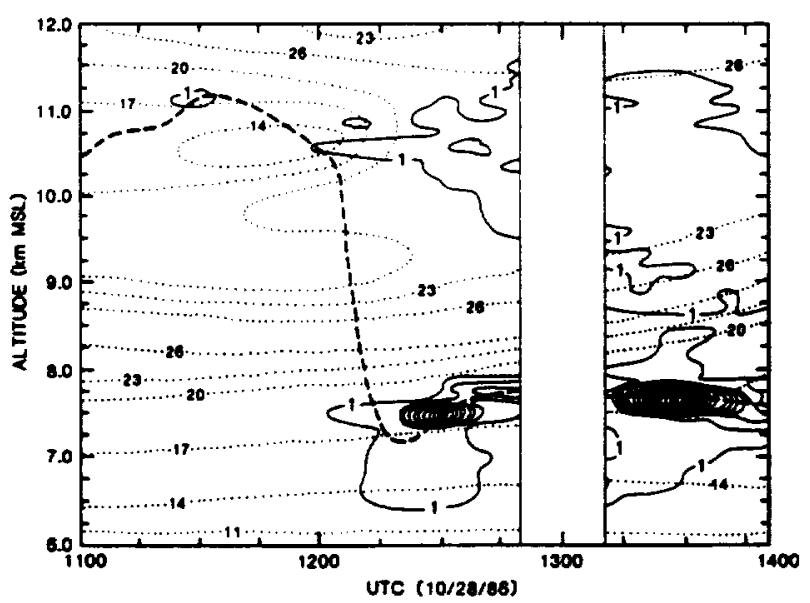

Fig. 8. Time height backscatter cross section $\left(10^{-3} \mathrm{~km}^{-1} \mathrm{sr}^{-1}\right.$, interval: $\left.7(10)^{-3} \mathrm{~km}^{-1} \mathrm{sr}^{-1}\right)$ of a two-layer cirrus formation with altocumulus embedded in the lower layer. The $I(10)^{-3} \mathrm{~km}^{-1} \mathrm{sr}^{-1}$ contour near the ACu outlines what appears to be ice virga. The largest backscatter cross section observed during the case study $(0.18$ $\mathrm{km}^{-1} \mathrm{sr}^{-1}$ ) occurred $\sim 1330$ UTC at $\sim 7.6 \mathrm{~km}$. A table of localized backscatter phase function and optical thickness illustrating the differences in optical properties of $\mathrm{ACu}$. ice virga, and the upper cloud layer may be found in Sassen et al. (1990). Isotachs are plotted in dotted line in $\mathrm{m} \mathrm{s}^{-1}$ from the Forth McCoy radiosondes launched at 0900,1200 , and 1500 UTC.

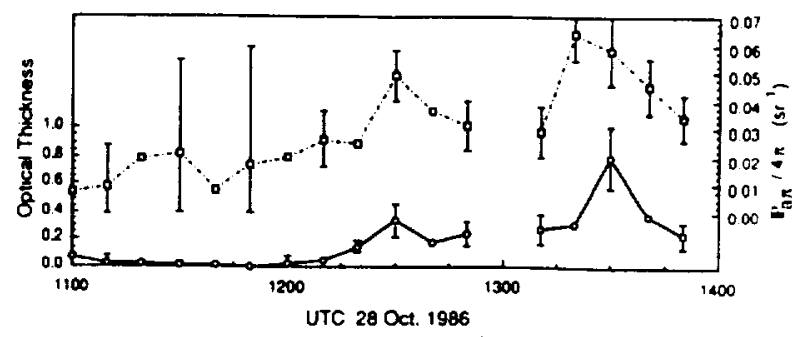

FIG. 9. Time history of the total cloud optical depth (-) and bulk normalized backscatter phase function $\left(-\cdots, \mathbf{s r}^{-1}\right)$ coincident with the backscatter cross sections plotted in Fig. 8. Ten minute averaging has been applied (see section 3 ) to smooth the data. The largest values of backscatter phase function values in this record coincide with the cirrus regions containing altocumulus cells.

highlights the dissimilarity in lidar backscatter between these cloud types. The lower layer cloud appears to be formed from a series of relatively dense altocumulus cells, each with a $0.5-1.0 \mathrm{~km}$ long ice crystal fallout tail. The identification of these clouds as ACu is inferred from an all-sky photograph taken at Wausau and the fact that similar cloud structure was observed at Ft. McCoy and Wausau lidar data around this time period (Sassen et al. 1990). Ice virga is inferred from the environmental temperature $\left(-25^{\circ}\right.$ to $\left.-30^{\circ} \mathrm{C}\right)$ and from grey-scale images of lidar backscatter. Virga appears in these images as finely detailed striated sheets that deviate from vertical following the altitude profile of horizontal wind sheer. Translation with the wind speeds at $7.7 \mathrm{~km}$ would indicate the $\mathrm{ACu}$ were about $20 \mathrm{~km}$ across, yet they were $\sim 200 \mathrm{~m}$ thick, producing a height to width ratio of only $1: 100$. As the $\mathrm{ACu}$ developed, Fig. 9 shows an increase in both the total cloud optical thickness and the backscatter phase function, while the optical midcloud altitude decreased from $\sim 11 \mathrm{~km}$ to $\sim 7.5 \mathrm{~km}$. Note that the average cloud backscatter phase function peaks with the passage of each $A C u$ cell. Because similar cloud features were also observed to produce little depolarization at Wausau and $\mathrm{Ft}$. McCoy, we can infer, with some confidence, that the backscatter phase function peaks suggest these clouds are composed of supercooled spherical water droplets (see Sassen et al. 1990 for depolarization data and spatially localized backscatter phase functions).

The $\mathrm{ACu}$ bands at $\sim 7.6 \mathrm{~km}$ produce significantly greater backscatter than either the $9-12 \mathrm{~km}$ cirrus or the lower level virga between 6.4 and $7.1 \mathrm{~km}$. The $\beta_{a x} /$ $4 \pi$ maximum reached at 1419 exceeded $0.15 \mathrm{~km}^{-1}$ $\mathrm{sr}^{-1}$, nearly an order of magnitude greater than the largest $\beta_{a x} / 4 \pi$ observed during this case study in any cirrus exhibiting significant vertical development. One reason for this is that $\mathrm{ACu}$ are primarily composed of supercooled liquid water droplets; because of optical surface wave phenomena, such droplets are more efficient at backscattering than nonspherical ice crystals. A second reason is that supercooled water droplets are necessarily quite small; whereas, once glaciation begins. cloud particles accumulate mass rapidly because of the 
reduction of saturation vapor pressure with respect to ice. Hence, ice particle fall speeds rapidly exceed vertical atmospheric motion, and particle mass distributes over an extended column, decreasing the local backscatter intensity. The backscatter cross section will also diminish as growth processes redistribute water mass from many small particles to fewer but larger ice crystals with substantial fall speeds. This is because particle mass increases as the cube of the radius while the scattering cross section approximately follows the radius squared.

The $\mathrm{ACu}$, when present, dominate the optical thickness profile. This effect is evident in the dramatic decrease of $4 \mathrm{~km}$ in the optical midcloud altitude between 1200 and 1215. Clearly, the occurrence of $\mathrm{ACu}$ can have significant impact on the radiative properties of the cloud independent of cloud top altitude and bottom altitudes.

\section{d. Cirrostratus}

From 1500 UTC 28 October to the end of the case study period, the cirrus observed over Madison assumed a distinct pattern of vertically developed cells with regions of enhanced backscatter appearing near both cloud top and cloud bottom (see Fig. 10). The vertical elongation of backscatter cross section contours suggests an upward and/or downward redistribution of cloud particles. The top and bottom cellular morphology of this layer is most evident $\sim 1610$ where an apparent generating region near cloud top produces a

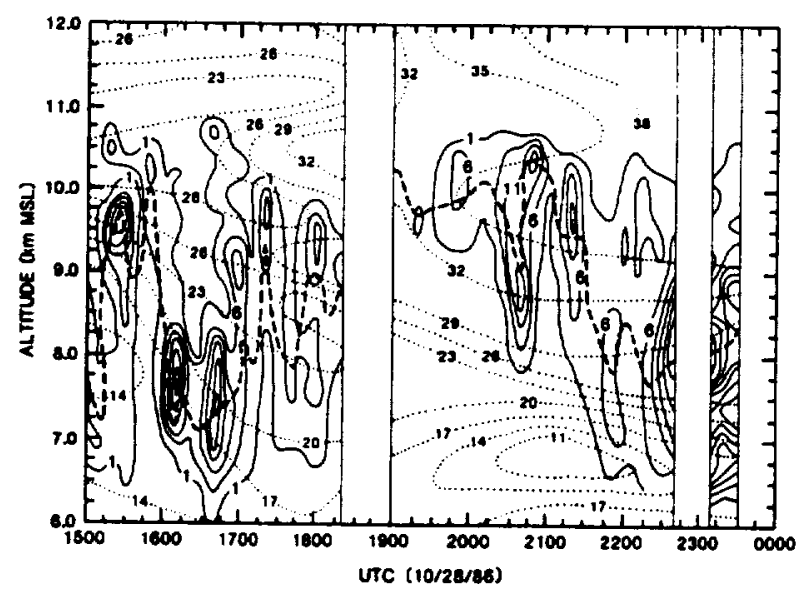

FIG. 10. From 1500 UTC until operations were terminated, a cirrostratus layer was observed over Madison which contained a distinct pattern of vertically developed cells evident in this time height backscatter cross section $\left(10^{-3} \mathrm{~km}^{-1} \mathrm{sr}^{-1},-\right)$ profile. Regions of intensified backscatter are found near both cloud top and cloud bottom. The optical midcloud height (- -) tends to follow these regions independent of cloud top and bottom altitudes. The relatively cloud free period $\sim 1900$ UTC corresponds to the clear arc region noted in satellite imagery by Starr and Wylie (1990). Isotachs (....) are plotted in $\mathrm{m} \mathrm{s}^{-1}$ determined by radiosondes launched from $\mathrm{Ft}$. McCoy at 1500,1800,2100, and 2400 UTC.

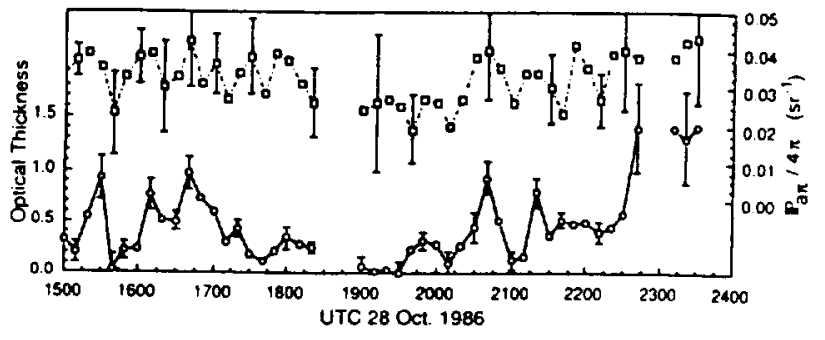

FIG. 11. Time history of the total c' und optical depth ( - ) and bulk normalized backscatter phase function $\left(-\cdot \cdot, \mathrm{sr}^{-1}\right)$ coincident with the backscatter cross sections plotted in Fig. 10. Peaks in the backscatter phase function tend to coincide with apparent generating regions while minima tend to occur between cells independent of cloud optical thickness suggesting different particle microphysics for the two regimes.

virga trail that extends to, and perhaps seeds, a second region of strong backscatter near $\sim 7.5 \mathrm{~km}$. The lower regions (at 1610 and 1640 ) also appear to be precipitating as evidenced by the vertical elongation of the cloud bottom backscatter cross section contours. Note that the midcloud altitude follows regions of enhanced backscatter very closely and that there is no obvious relationship between the appearance of these regions and either cloud top or cloud bottom altitudes. Thus, the structures that dominate cloud optical properties are not necessarily indicated by either the cloud boundaries or the physical midcloud altitude.

With the exception of two short periods, near 1540 and 1915, the cirrus optical thickness exceeded 0.1 forming an apparent cirrostratus layer (see Fig. 11). The gap in cloud cover at $\sim 1900$ corresponds to the passage of the clear arc identified from satellite imagery in Starr and Wylie (1990). Note that the peaks in backscatter-phase function $\left(\sim 0.038 \mathrm{sr}^{-1}\right)$ tend to coincide with the occurrence of the regions of enhanced backscatter cross section independent of total optical thickness, while the regions between the cells usually produce the minima in the backscatter phase function values of $\sim 0.02-0.026 \mathrm{sr}^{-1}$. This suggests differences in the microphysical properties for the particles associated with the cells compared with those of the surrounding cloud. One explanation may be that the lower regions of enhanced backscatter are areas of cloud particle generation that may contain an abundance of small supercooled water droplets (temperature at 7.5 $\mathrm{km}: \sim-30^{\circ} \mathrm{C}$; at $9.5: \sim-45^{\circ} \mathrm{C}$ ). Although the generation mechanism is unclear, an abundance of exceedingly small particles could also account for the observed backscatter-phase function effects (as well as low depolarization ratios, see Sassen et al. 1990). This issue cannot be resolved from in situ probe data because present instruments are incapable of resolving such small particles. However, Heymsfield et al. (1990) report observing clouds containing supercooled water droplets using aircraft borne in situ probes. In regions between the cells, it may be that sufficient time has elapsed so that the number density of droplets has been 
substantially diminished by differential vapor pressure effects between ice and water, or the smallest of particles may have grown by some coalescence phenomena.

The modulation of the bulk backscatter-phase function also suggests that some of the apparent structure observed in the backscatter cross section plots is generated by microphysical changes and is not simply proportional to the distribution of cloud extinction cross section. This effect should be considered when deducing cloud morphological and optical characteristics from simple plots of lidar backscatter.

The backscatter contours in Fig. 10 indicate a large scale pattern of intensification with a period of about 45 minutes. However, an examination of the higher time resolution pictures of the raw laser returns reveals the clouds are composed of many repeated finer scale features. Figure 12 is an expanded scale view of the 1800 to 2230 time period that shows a striated $5 \mathrm{~min}$ structure imbedded in the larger scale organization. This pattern is most clearly developed between 2130 to 2230. Wind drift estimates would suggest that these features have a horizontal scale of about $9-11 \mathrm{~km}$; thus, the height to width aspect ratio of the elementary structures is $\sim 1: 5$; whereas the larger scale field of enhanced backscatter exhibits an aspect ratio of $\sim 1: 25$.

\section{Summary}

The HSRL has been successfully adapted to the task of cirrus cloud optical property measurement. The transmitter configuration used to acquire the case study data produce $\sim 50 \mathrm{~mW}$ of output power and achieved eye-safe, direct optical depth, and backscatter cross section measurements with 10 min averaging times. A new continuously pumped, injection seeded, frequency doubled Nd:YAG laser transmitter has recently reduced time-averaging constraints by a factor of $\sim 10$, while improving the aerosol-molecular signal separation capabilities and wavelength stability of the instrument (Grund and Eloranta 1990).

The cirrus cloud backscatter cross sections, optical thickness, and backscatter-phase functions have been determined for the 27-28 October 1986 segment of the HSRL FIRE dataset. Features exhibiting backscatter cross sections ranging over four orders of magnitude have been observed within this 33 hour period. Extended episodes of low backscatter cross section $\left(\sim 10^{-7} \mathrm{~m}^{-1} \mathrm{sr}^{-1}\right)$ have been observed between structured cirrus clouds events.

During this period, cirrus clouds were observed with optical thicknesses ranging from 0.01 to 1.4 . Bulk-av-

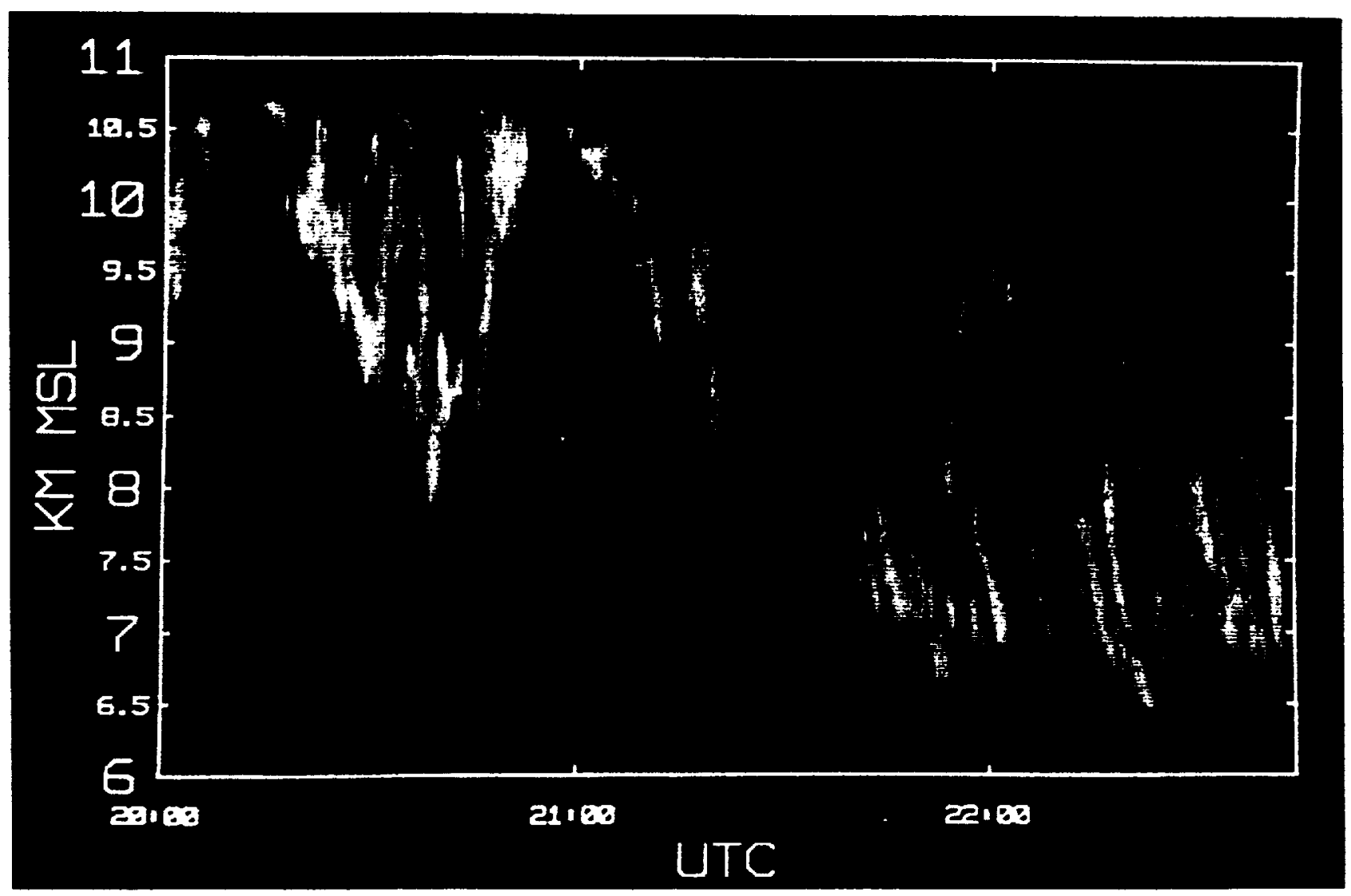

FIG. 12. Intensity modulated height time display of the $\log$ of the $R^{2}$ corrected HSRL aerosol channel signal. The image has 10 times the temporal resolution necessary to produce the calibrated attenuation corrected cross section plotted in Fig. 10. The image reveals an $\sim 5$ minute structure $(\sim 9-11 \mathrm{~km}$ wide) imbedded in the larger scale organization shown in Fig. 10. 
erage backscatter-phase functions in the range of 0.02 $0.065 \mathrm{sr}^{-1}$ were also observed. A variety of cirrus cloud structures were observed with apparent sizes ranging from 5 to $400 \mathrm{~km}$ during this period that exhibited aspect ratios of from $1: 5$ to $1: 1$.

The altitude relationship between cloud top and bottom boundaries and the optical center of the cloud is influenced by the type of formation observed. $\mathrm{ACu}$ and uncinus generating regions tend to concentrate attenuation in regions of less than $200 \mathrm{~m}$ thickness that dominate the extinction profile, even when the generating cell caps an extended column of virga. Virga exhibits complicated fine scale structure, often lying in interleaved, sheared sheets. In cirrostratus, imbedded vertically-developed cells frequently occupy a significant altitude range, and create large spatial inhomogeneities in optical properties.

Cirrus morphology and generation processes appear to be related to the wind field, probably responding to secondary circulations. Better temporal and spatial resolution in wind measurements in future experiments would aid the understanding of cirrus generation and dissipation mechanisms.

Acknowledgments. We wish to thank Dr. D. O'C. Starr of NASA Goddard Space Flight Center for providing us with an analysis of the radiosonde data and Dr. D. P. Wylie of the University of Wisconsin for providing the GOES satellite images. Discussions with both Dr.'s Starr and Wylie proved invaluable in determining the meteorological processes related to the cirrus observations in this case study.

We also thank Daniel Forrest, Scott Kohn, and Sevtap Karakoy-Otles for providing important data analysis and programming support for this project.

Funding for HSRL development, data acquisition and the collection of cloud statistics has been provided under ARO Grant DAAG29-84-K-0069 and ONR Contract N00014-87-K-0436. Recent HSRL algorithm development, the incorporation of Brillouin scattering corrections in the molecular scattering model used to calibrate this data, and studies of the correlations between wind and backscatter cross section have been funded under AFGL contract FI 9628-87-0056. Analysis of HSRL FIRE related data is partially supported under NASA Grant NAG1-882.

\section{APPENDIX}

\section{HSRL Theory}

The basic principles inherent to all monostatic lidar systems are embodied in the lidar equation:

$$
\begin{array}{r}
P(R)=E_{0} \xi R^{-2}\left[\beta_{a}(R) \frac{\mathbf{P}_{a}(\pi, R)}{4 \pi}+\beta_{m}(R) \frac{\mathbf{P}_{m}(\pi)}{4 \pi}\right] \\
\times \exp \left[-2 \int_{0}^{R} \beta_{c}\left(r^{\prime}\right) d r^{\prime}\right]+M(R)
\end{array}
$$

(after Collis and Russel 1976).
Here, $P(R)$ is the power incident upon the receiver from range $R, E_{0}$ is the energy of the transmitted pulse. $\xi=A_{r}(c / 2)$ where $A_{r}$ is the receiver area and $c$ is the speed of light. The subscripts " $a$ " and " $m$ " denote aerosol and molecular scattering quantities. The term aerosol includes both small aerosol and cirrus cloud particles. $\beta_{a}(R)$ and $\beta_{m}(R)$ are the respective scattering cross sections per unit volume, $P_{a}(\pi, R) / 4 \pi$ and $P_{m}(\pi) / 4 \pi$ are the respective normalized backscatterphase functions, $\beta_{c}(R)$ is the extinction cross section per unit volume, and $M(R)$ is the contribution from multiple scattering. The entire expression in the first set of brackets is the total normalized backscatter cross section per unit volume, $\beta_{x}(R)$. Both $\beta_{x}(R)$ and $\beta_{e}(R)$ are due to the effects of aerosols and molecules. The exponential term accounts for the two-way optical thickness between the lidar and the backscattering volume, where the optical thickness accumulated between range $R_{1}$ and $R_{2}$ is represented as:

$$
r=\int_{R_{1}}^{R_{2}} \beta_{c}\left(r^{\prime}\right) d r^{\prime} .
$$

Equation (A1) demonstrates that the lidar return depends upon both the local value of $\beta_{\pi}(R)$ and upon $\tau$ between the lidar and $R$. Only a single measurement of $P(R)$ is provided at each range by single-channel lidar systems, leading to ambiguities in the direct evaluation of $\beta_{z}$ or $\beta_{\varepsilon}$. In fact, there are an infinite family of $\beta_{c}(R)$ profiles for which an increase in optical thickness with penetration depth is just cancelled by an increase in backscatter with range, rendering the medium invisible to single-channel lidar systems of low sensitivity (Grund and Eloranta 1987). In optically thicker cirrus clouds, the multiple scattering contribution $M(R)$ can further complicate matters by effectively increasing $P(R)$ in a way that depends upon the unknown spatial distributions of the angular scattering phase function and optical thickness between the lidar and the sensed volume (Eloranta and Shipley 1982).

As mentioned, several techniques have been employed to untangle $\beta_{\varepsilon}$ from $\beta_{t}$. Each method requires significant assumptions about the distribution of scatterers and about the nature and profile of the particle backscatter to extinction ratio. The multiple scattering contribution, which can become large in returns from cirnus clouds, may be minimized by a narrow field of view (fov) design for the lidar system. This solution is often difficult to achieve and is therefore not frequently implemented; however, narrow fov is a necessary requirement for the high resolution spectrometer employed in the HSRL (see Table 1), thus the uncertainties caused by the effects of multiple scattering processes are greatly reduced in the data acquired with this system (Grund 1987).

Because the HSRL separately measures molecular and particulate backscatter, two lidar equations may be written that are coupled by the common unknown integral of extinction. Assuming $M(R)$ is negligible, 
the molecular and particulate lidar equations may be written:

$$
P_{m}(R) R^{2}=E_{0} \xi \beta_{m}(R) \frac{3}{8 \pi} \exp \left[-2 \int_{0}^{R} \beta_{a}\left(r^{\prime}\right) d r^{\prime}\right]
$$

and

$$
\begin{aligned}
P_{a}(R) R^{2}=E_{0} \xi \beta_{a}(R) & \frac{\mathbf{P}_{a}(\pi, R)}{4 \pi} \\
& \exp \left[-2 \int_{0}^{R} \beta_{c}\left(r^{\prime}\right) d r^{\prime}\right]
\end{aligned}
$$

where the normalized molecular backscatter phase function $\left(\boldsymbol{P}_{m}(\pi) / 4 \pi\right)$ has been replaced with its analytic value, $3 / 8 \pi$.

Because the molecular scattering cross section $\left(\beta_{m}\right)$ is determined by the profile of atmospheric density calculated from a convenient radiosonde (or from climatology), (A3) is completely defined, and may be solved explicitly for the extinction $\beta_{t}$ from the measured lidar return $P_{m}(R)$. The ambiguity in separating extinction from backscatter in single-channel lidar retrievals is eliminated by the HSRL technique since the system is effectively calibrated at each range with the return from the known molecular backscatter target.

The aerosol backscatter cross section is unambiguously determined from the ratio of (A4) to (A3):

$$
\beta_{a}(R) \frac{\mathbb{P}_{a}(\pi, R)}{4 \pi}=\beta_{m}(R) \frac{3}{8 \pi} \frac{P_{a}(R)}{P_{m}(R)} .
$$

By solving ( $A 3$ ), the extinction cross section may be directly determined from the slope of the molecular backscatter return and a profile of molecular backscatter cross section calculated from the in situ density:

$\beta_{t}(R)=-\frac{1}{2}\left[\frac{d \ln \left(P_{m}(R) R^{2}\right)}{d R}-\frac{d \ln \left(\beta_{m}(R)\right)}{d R}\right]$.

(A6)

In the absence of aerosol and gaseous absorption, $\beta_{a}$ $=\beta_{c}-\beta_{m} ;$ therefore, the normalized backscatter phase function is uniquely determined from (A5) and the atmospheric density profile:

$$
\frac{\mathbf{P}_{a}(\pi, R)}{4 \pi}=\beta_{m}(R) \frac{3}{8 \pi} \frac{P_{a}(R)}{\left[\beta_{c}(R)-\beta_{m}(R)\right] P_{m}(R)} .
$$

\section{REFERENCES}

Collis, R. T. H., and P. B. Russell. 1976: Lidar measurement of particles and gases by elastic backscattering and differential absorption. Laser Monitoring of the Atmosphere. Springer-Verlag. $71-151$.
Eloranta, E. W., and S. T. Shipley, 1982: A Solution for Multiple Scattering. Atmospheric Aerosols: Their Formation. Optical Properties and Effects. Spectrum Press. 227-239.

- and D. K. Forrest, 1986: Generation of attenuation corrected images from lidar data. I Ith International Lidar Conf., Toronto. NASA Conference Pub. 2431, 3291-294.

Grund, C. J., 1987: Measurement of cirrus cloud optical properties by high spectral resolution lidar. Ph.D. thesis. University of Wisconsin-Madison, $92 \mathrm{pp}$.

- and E. W. Eloranta, 1987: Optically significant cirnus clouds may be rendered "invisible" to space-borne simple lidar systems. Opt. Soc. Amer. 1987 Technical Digest Series, 18, 75-78.

- and 1990: High repetition rate continuously pumped injection seeded Nd:YAG laser extends High Spectral Resolution Lidar capabilities. Op. Soc. Amer. 1990 Technical Digest Series. 4, 368-371.

- J. A. Weinman, and - 1990. Cirrus cloud velocity-backscatter correlations and instrument spatial resolution effects on remote wind sounding errors. Op. Soc. Amer. 1990 Technical Digest Series. 4, 158-161.

Klett. J. D., 1981: Stable analytic inversion solution for processing lidar returns. App. Opt., $20,211-220$.

Evans, W. E., E. J. Weigman, W. Viezee and M. G. H. Ligda, 1964: Performance specifications for a meteorological satellite lidar. NASA Report CR-760871, NTIS N66-2997, 174 pp.

Heymsfield, A. J., K. M. Miller and J. D. Spinhime. 1990: The 2728 October 1986 FIRE IFO cirrus case study: Cloud structure and composition from in situ measurements. Mon. Wea. Rev. 118 2313-2328.

Milton, M. J. T., and P. T. Woods, 1987: Pulse averaging methods for a laser remote monitoring system using atmospheric back scatter. App. Opt., 26, 2598-2603.

Platt, C. M. R., J. C. Scott and A. C. Dilley, 1987: Remote sounding of high clouds. Pan VI: Optical properties of midlatitude and tropical cirrus. J. Atmos. Sci., 44, 729-747.

Sassen, K., M. K. Giffin, and G. C. Dodd, 1989: Optical scattering and microphysical properties of subvisual cirrus clouds and climatic implications. J. Appl. Meteor., 28, 91-98.

- C. J. Grund, J. D. Spinhime, J. M. Alvarez and M. J. Hardesty, 1990: The 27-28 October 1986 FIRE IFO cirrus case study: a five lidar overview of cloud structure and evolution. Mon. Wea. Rev., 118, 2288-2312.

Shipley, S. T., D. H. Tracy, E. W. Eloranta, J. T. Trauger, J. T. Sroga, F. L. Roesler and J. A. Weinman, 1983: High spectral resolution lidar to measure optical scattering properties of atmospheric aerosols. 1: Theory and instrumentation. App. Opt., 22, 37163724.

Spinhirne, J. D., J. A. Reagan and B. M. Herman, 1980: Vertical distribution of aerosol extinction cross section and inference of aerosol imaginary index in the troposphere by lidar technique. J. Appl. Meleor., 19, 426-438.

Sroga, J. T., E. W. Eloranta, S. T. Shipley, F. L. Roesler and P. J. Tryon, 1983: High spectral resolution lidar to measure optical scattering properties of atmospheric aerosols. 2: Calibration and analysis. App. Opt., 22, 3725-3732.

Starr, D. O'C., 1987: A cirrus-cloud experiment: intensive field observations planned for FIRE. Bull. Amer. Meteor. Soc. 68, $119-$ 124.

-, and D. P. Wylie, 1990: The 27-28 October 1986 FIRE IFO cirnus cloud study: meteorology and clouds. Mon. Wea. Rev.
$118,2259-2287$.

Weinman, J. A., 1984: Tomographic lidar to measure extinction coef. ficients of atmospheric aerosols. App. Opt., 23, 3882-3888.

- 1988: The derivation of atmospheric extinction profiles and wind speed over the ocean from a satellite borne lidar. App. Opt., 27, 3994-4001.

Wylie, D. P., and W. P. Menzel, 1989: Two years of cloud cover statistics using VAS. J. Climate. 2, 380-392.

Yip. S., and M. Nelkin, 1964: Application of a kinetic model to timedependent density correlations in fluids. Phys. Rev., 135, A 1241-
A 1247 . 